\title{
Quasi-Fermi Levels, Chemical and Electric Potential Profiles of a Semiconductor Under Illumination
}

\author{
A. M. dos Santos, D. Beliaev, L. M. R. Scolfaro, and J. R. Leite \\ Instituto de Física da Universidade de São Paulo, \\ Caixa Postal 66318, CEP 05315-970, São Paulo, SP, Brazil
}

Received February, 1999

\begin{abstract}
A theoretical semi-classical method to calculate the modulated field profile in a semiconductor is presented. The behavior of quasi-Fermi levels and chemical potentials for the majority and minority carriers is investigated. Systematic studies of the non-thermodynamic equilibrium electric potential profile as a function of the modulation light intensity are performed.
\end{abstract}

While the methodology used in the determination of the non-modulated field spatial distribution in a semiconductor is easily found in literature $[1,2,3,4]$, the same does not happen when a treatment of a system under influence of modulating agents, that is, at the nonthermodynamic equilibrium, is required. The problem of determining the electronic band spatial profile and of investigating the behavior of quasi-Fermi levels at the non-thermodynamic equilibrium was already approached semi-empirically by different authors. [5, 6, 7] However, these studies are limited to the flat quasiFermi level approximation [5, 6] and to the assumption of electronic bands edges, parabolic in real space. $[5,6,7]$ An attempt to consider a more general case with non-flat quasi-Fermi levels in a depletion region was made, but the shape of the quasi-Fermi levels in this region was arbitrarily drawn. [7] The necessity to calculate modulated electric field profiles for a rigorous determination of modulation spectroscopy lineshapes is urged to interprete a wide number of experimental optical studies. [3, 4, 8, 9, 10] Thus, a method to obtain the modulated profiles of the electric potential and field is highly desirable.

We calculate the band bending profile selfconsistently following the approach for the nonmodulated electric field profile developed earlier. [1, 2, $3,4]$ The importance of the self-consistency in such kind of calculations was also pointed out recently. [4, 11] A bulk semiconductor extends from $z=0$ to $z=\infty$. The other infinite half space is assumed to be a vacuum. The potential $\varphi(z, I)$ of a semiconductor modulated with light intensity $I$ depends on the total charge density $\rho(z, I)$ and is governed by the integral Poisson's equation [12]

$$
\varphi(z, I)=\varphi(0, I)+\frac{4 \pi}{\varepsilon} \int_{\infty}^{z} d z^{\prime} \int_{\infty}^{z^{\prime}} \rho\left(z^{\prime \prime}, I\right) d z^{\prime \prime},
$$

There are two essential differences to be noted in comparison to the non-modulated case. [1, 2, 3, 4] First, the surface barrier $\varphi(0, I)$ depends on the modulating light intensity $I$. Second, the total charge density

$$
\rho(z, I)=-e\left[n(z, I)+N_{A}^{-}(z, I)-p(z, I)-N_{D}^{+}(z, I)\right]
$$

depends on the chemical potentials for electrons $\left(\xi_{n}\right)$ and holes $\left(\xi_{p}\right)$ through $n, p, N_{A}^{-}, N_{D}^{+}$, the electron, hole, ionized acceptor and ionized donor concentrations, respectively, calculated through Fermi statistics. Hence, Eq.(2) can describe degenerated and non-degenerated cases. If a stationary state is achieved, the remaining difference between these chemical potentials implies an equilibrium in the energy distribution of the carriers inside the electron and valence bands but not between carriers from different bands. This consideration is justified by a low intra-band energy relaxation time when compared to the lifetime of the generated carriers. [13] In this way, we can say that our perturbed sample finds itself in the state of stationary non-thermodynamic equilibrium. By analogy to the case of thermodynamic statistics, in standard expressions for $n, p, N_{A}^{-}, N_{D}^{+}$we substitute the thermodynamic equilibrium chemical potential $\xi$ by $\xi_{n}$ for electrons and by $\xi_{p}$ for holes. Using the continuity equation and equalling the photogenerated current density (depending on the generation $G$ and recombination $R$ ) to the sum of the diffusion and drift current densities we obtain quasi-Fermi levels in terms of the photoinduced current 


$$
\begin{aligned}
& E_{F n}(z, I)=E_{F}-e^{2} \int_{\infty}^{z} \sigma_{n}^{-1}\left(z^{\prime}, I\right) d z^{\prime} \int_{\infty}^{z^{\prime}}\left(G_{n}\left(z^{\prime \prime}, I\right)-R_{n}\left(z^{\prime \prime}, I\right)\right) d z^{\prime \prime} \\
& E_{F p}(z, I)=E_{F}+e^{2} \int_{\infty}^{z} \sigma_{p}^{-1}\left(z^{\prime}, I\right) d z^{\prime} \int_{\infty}^{z^{\prime}}\left(G_{p}\left(z^{\prime \prime}, I\right)-R_{p}\left(z^{\prime \prime}, I\right)\right) d z^{\prime \prime}
\end{aligned}
$$

where $E_{F}$ stays for the Fermi level energy at the thermodynamic equilibrium and $\sigma_{n}$ and $\sigma_{p}$ are the electron and hole conductivities, respectively. To deduce Eqs (3) and (4) we assume the non-degenerated form of the equation for current density. Using $\xi_{n}(z, I)=e \varphi(z, I)+E_{F n}(z, I), \xi_{p}(z, I)=e \varphi(z, I)+E_{F p}(z, I)$, and Eq.(1) we obtain two coupled equations for the chemical potentials

$$
\begin{aligned}
& \xi_{n}(z)=\frac{4 \pi e}{\varepsilon} \int_{\infty}^{0} d z^{\prime} \int_{\infty}^{z^{\prime}} \rho\left(z^{\prime \prime}\right) d z^{\prime \prime}+\xi_{n}(0)+e^{2} \int_{\infty}^{z} \sigma_{n}^{-1}\left(z^{\prime}\right) d z^{\prime} \int_{\infty}^{z^{\prime}}\left(G_{n}\left(z^{\prime \prime}\right)-R_{n}\left(z^{\prime \prime}\right)\right) d z^{\prime \prime} \\
& \xi_{p}(z)=\frac{4 \pi e}{\varepsilon} \int_{\infty}^{0} d z^{\prime} \int_{\infty}^{z^{\prime}} \rho\left(z^{\prime \prime}\right) d z^{\prime \prime}+\xi_{p}(0)-e^{2} \int_{\infty}^{z} \sigma_{p}^{-1}\left(z^{\prime}\right) d z^{\prime} \int_{\infty}^{z^{\prime}}\left(G_{p}\left(z^{\prime \prime}\right)-R_{p}\left(z^{\prime \prime}\right)\right) d z^{\prime \prime}
\end{aligned}
$$

where $\xi_{n}(z)=\xi_{n}(z, I)$, etc... . These expressions are coupled since each of the potentials is dependent not only on its own value but on the value of the chemical potential for the other type of carriers too. Since Eqs.(5) and (6) are unlikely to have analytical solutions, they should be solved self-consitently taking into consideration their coupling.

In semiconductors the photogeneration of electronhole pairs predominates over the generation of single carriers, then we write $G_{n}(z)=G_{p}(z)=G(z)$ and

$$
G(z)=\left(\frac{\alpha I e^{-\alpha z}}{\omega}\right)
$$

$\omega$ is the modulating photon frequency, and $\alpha$ stays for the absorption coefficient. [14] In the case of stationary non-thermodynamic equilibrium state the electron and hole recombinations should be the same at any chosen point and are represented by a Shockley-Read-Hall recombination [15]. Thus, $R_{n}(z)=R_{p}(z)=R(z)$ and

$$
R(z)=\frac{n(z) p(z)-n_{0}(z) p_{0}(z)}{\tau_{p 0}\left(n(z)+n_{t}(z)\right)+\tau_{n 0}\left(p(z)+p_{t}(z)\right)}
$$

Here, $\tau_{n(p) 0}=s_{n(p)}^{-1} \times v_{n(p)}^{-1} \times N_{t}^{-1}$ stays for the electron (hole) capture time; $s_{n(p)}$ is the electron (hole) capture cross section; $v_{n(p)}$ is the electron (hole) thermal velocity.
In order to find solutions for the Eqs.(5) and (6), we need to determine the surface value of the chemical potential for majority carriers, either $\xi_{n}(0)$ or $\xi_{p}(0)$, depending on the $n$ - or $p$-type of the surface layer. As the density of the majority carriers is elevated in comparison to the minority carriers density, one could suppose that the majority carriers are the main agents responsible for the change of the electric potential. At the surface the difference between the modulated and nonmodulated electric potential values depends on the photoinduced current density $j[10]$

$$
\varphi(0, I)-\varphi(0,0)=\Delta \varphi(0)=\frac{\eta k_{B} T}{e} \ln \left(\frac{j}{j_{\text {sat }} \cdot r}+1\right)
$$

where $j_{\text {sat }}$ is the non-perturbed saturation current density; $\eta$ is so-called ideal factor and $r$ stays for the ratio between the surface area contributing to the saturation current and the illuminated surface area. The above equation could be used as a boundary condition for the chemical potential of the majority carriers if one equals the surface electric potential value determined by Eq.(9) to its value coming out of the solution of Eqs.(5) and (6). It is worth noting that $j$ is a functional of $\xi_{n}(z)$ and $\xi_{p}(z)$. It must be calculated at each iteration during the solution Eqs.(5) and (6), providing a self-consistently adjusted use of this quantity as a boundary condition via Eq.(9). 
As an example, we consider the chemical potentials in $n$-type $\left(N_{D}=5 \times 10^{16} \mathrm{~cm}^{-3}\right.$, non-degenerated case) bulk GaAs subject to illumination, $\mathrm{T}=300 \mathrm{~K}$. In our calculations we used the following values of the electron and hole mobilities and capture cross sections: $\mu_{n}=8 \times 10^{3} \mathrm{~cm}^{2} V^{-1} s^{-1}, \mu_{p}=4 \times 10^{2} \mathrm{~cm}^{2} V^{-1} s^{-1}$ [16], $s_{n}=10^{-17} \mathrm{~cm}^{2}, s_{p}=10^{-16} \mathrm{~cm}^{2}$ [12], respectively. The deep centers concentration was assumed $N_{t}=10^{12} \mathrm{~cm}^{-3}$. In Fig.1 we present calculated profiles of the chemical potentials as a function of the light intensity. One could observe that for low light intensities, the system tends to the thermodynamic equilibrium since the modulated chemical potentials approach the non-modulated curve. For the high light intensities the minority carrier chemical potential shows a practically constant behavior. This is due to the almost complete flattening of the valence and conduction band edges. At the same intensity the majority carrier chemical potential tends also to a flat behavior. Fig. 1 clearly shows that for the high modulating light intensities the minority carrier chemical potential at the surface drops below its thermodynamic equilibrium value. This contradicts the well known and widely employed expression linking the difference between quasi-Fermi levels at an illuminated semiconductor surface and the photoinduced change in the surface potential: $[5,6]$

$$
-e \Delta \varphi(0)=E_{F n}-E_{F p}
$$

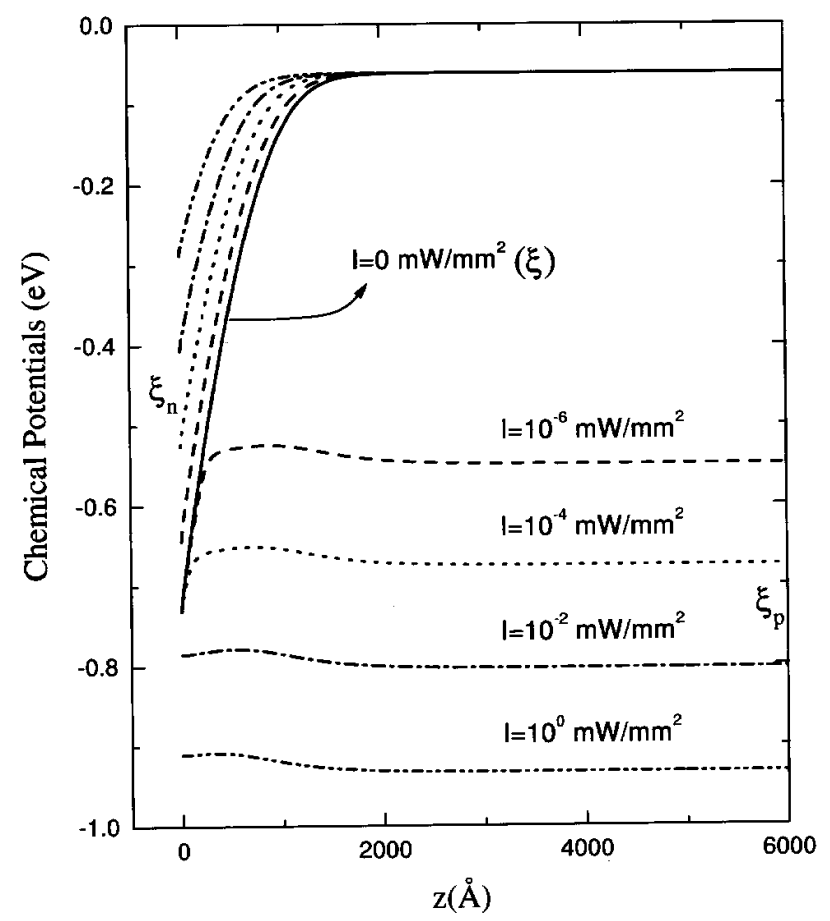

Figure 1. Calculated chemical potentials for electrons $\left(\xi_{n}\right)$ and holes $\left(\xi_{p}\right)$ as a function of the modulating light intensity $I$ for $n$-type bulk $G a A s$. Zero on the ordinate coincides with the conduction band bottom.
This condition is an implicit assumption that at the surface the minority carrier chemical potential has always the same value of the non-modulated case. Thus, our self-consistent calculations reveal that this expression is applicable only for low modulating light intensities.

Fig. 2 depicts calculated quasi-Fermi levels profiles and the conduction and valence band edges for the $p$ type bulk GaAs with dopant concentrations equal to $5 \times 10^{16} \mathrm{~cm}^{-3}$. We observe that the behavior of the quasi-Fermi level for the majority carriers (holes) practically does not change, continuing flat even under pertubation. Note that this comes as a result of our selfconsistent calculations and not as an assumption as in semi-empirical approaches. $[5,6,7]$ The values of the hole quasi-Fermi level for any light intensity is very similar to the Fermi level value in the non-perturbed material; however, changes are seen in the band edges profiles. For the non-modulated system the free carrier current equals to zero due to the anihilation of the drift current by the diffusion current. Since the Fermi level at the surface is pinned in the middle of the gap, the hole density in the depletion region is rather low. Under illumination the electric potential decreases, inducing a decrease of the electric field strength. The diffusion current overcomes the drift current causing the increase of the hole density close to the surface.

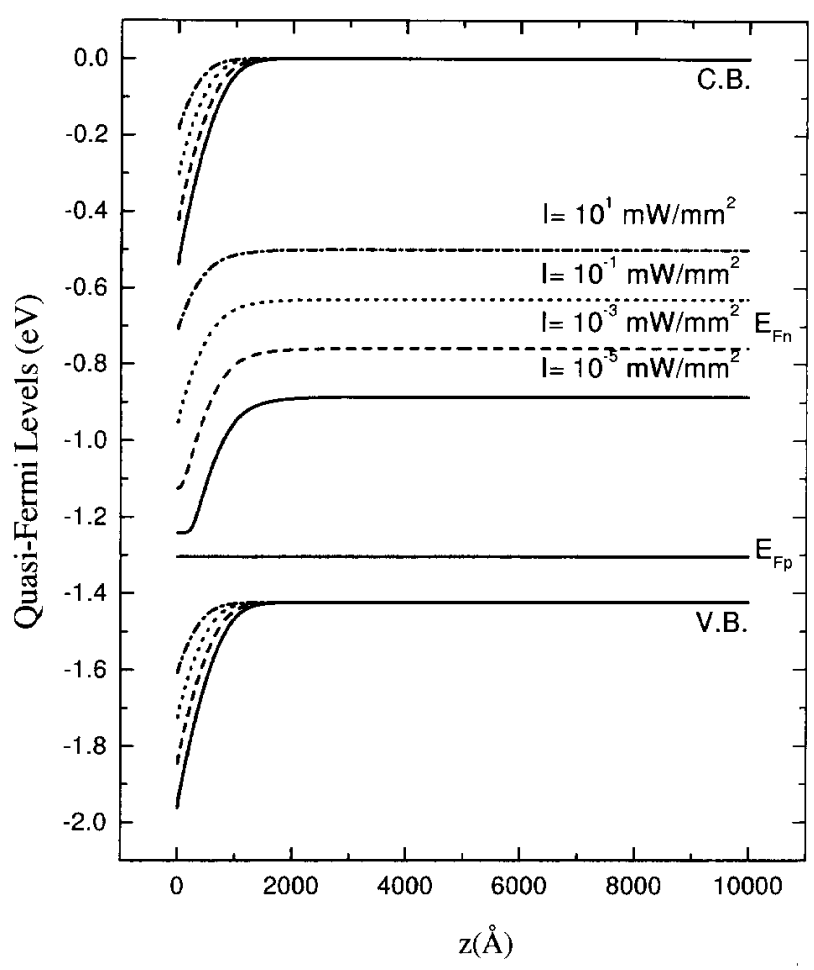

Figure 2. Calculated conduction (C.B.) and valence band (V.B.) edges and quasi-Fermi levels for different modulating light intensities in bulk GaAs of $p$-type. 
For the minority carriers (electrons) quasi-Fermi levels, one could clearly see the increase of the energy separation from the unperturbed Fermi-levels with the increase of the light intensity. Under low light intensities the number of electrons near the surface remains practically unchanged. This coincides with the practically flat behavior of the electron quasi-Fermi level in the proximity of the surface. For higher light intensities this behavior disapears. This means that the perturbation is already sufficient to modify the electron density not only far in the bulk but also at the surface. For the electrons the changes in their charge density are mostly due to the photogeneration and not to the drift and diffusion currents, as in the case of holes, since the decrease in the surface potential leads to a decrease in the number of electrons, present in the depletion region. Under the flat band conditions the electron quasi-Fermi level reaches the flat behavior near the edge of the conduction band. It should be noted that far in the sample for the whole range of light intensities it approaches the hole quasi-Fermi level, that is, the Fermi-level of the unperturbed material.

In summary, the behavior of the chemical potentials and quasi-Fermi levels was investigated for the stationary but non-equilibrium state of a semiconductor for different light intensities. Applications of our method to photoreflectance spectra calculations are in progress and will be reported elsewhere. [12]

The authors thank CNPq, FAPESP, FINEP, and CAPES for partial financial support.

\section{References}

[1] D. Beliaev, R. Enderlein, J. A. N. T. Soares, L. M.
R. Scolfaro, A. Marti Ceschin, A. A. Quivy, and J. R. Leite, Superlattices and Microstructures, 15, 339 (1994).

[2] R. Enderlein, D. Beliaev, J. A. N. T. Soares, L. M. R. Scolfaro, and J. R. Leite, Phys. Rev. B 52, 2814 (1995).

[3] J. A. N. T. Soares, D. Beliaev, R. Enderlein, L. M. R. Scolfaro, M. Saito and J. R. Leite, Material Science and engineering B 35, 267 (1995).

[4] J. A. N. T. Soares, R. Enderlein, D. Beliaev, J. R. Leite, and M. Saito, Semicond. Sci. Technol. 13, 1418 (1998); N. M. Sotomayor Choque, D. Beliaev, J. A. N. T. Soares, L. M. R. Scolfaro, A. L. Sperandio, A. A. Quivy, and J. R. Leite, to be published.

[5] P. Papayotatos and H. C. Card, Solid-St. Electron. 23, 41 (1980).

[6] Ching-Lang Chiang and Sigurd Wagner, IEEE Trans. Electron Dev. 32, 1722 (1985).

[7] Cheng-Lyang Jang, Solid-St. Electron. 34, 1613 (1991).

[8] D. Yan, Fred H. Pollak, T. P. Chin and J. M. Woodall, Phys. Rev. B. 52, 4674 (1995).

[9] J. S. Hwang, W. Y. Chou, and S. L. Tyan, H. H. Lin and T. L. Lee, Appl. Phys. Lett. 67, 2350 (1995).

[10] H. Shen and M. Dutta, J. Appl. Phys. 78, 2151 (1995).

[11] A. M. Yafyasov and I. M. Ivankiv, Phys. Stat. Solidi (b) 208, 41 (1998).

[12] A. M. dos Santos, D. Beliaev, L. M. R. Scolfaro, and J. R. Leite, to be published.

[13] K. Hess, "Advanced Theory of Semiconductor Devices", N. J. Englewood Cliffs, Printice-Hall (1988).

[14] D. R. Frankl "Electrical Properties of Semiconductor Surfaces", Oxford, New York, Pergamon Press (1967).

[15] S. C. Choo, L. S. Tan, and A. H. See, Solid-St. Electron. 36, 989 (1993).

[16] S. M. Sze, "Physics of semiconductor devices", New York, Saunder College Publishing (1981). 